\title{
Unilateral vocal cord paralysis following a short period of endotracheal intubation anesthesia
}

\author{
Yeun Hee Shin, Dong Ai An, Won Joon Choi, and Yun Hong Kim
}

Department of Anesthesiology and Pain Medicine, Kangbuk Samsung Hospital, Sungkyunkwan University School of Medicine, Seoul, Korea

Endotracheal intubation is a relatively safe procedure during general anesthesia. Nonetheless, many complications can occur after endotracheal intubation, among which sore throat and hoarseness are the most common symptoms [1]. We experienced hoarseness and aspiration of food due to vocal cord paralysis after a 150 minute laparoscopic nephrectomy.

An 80-year-old woman at $145 \mathrm{~cm}$ and $58 \mathrm{~kg}$ was admitted to the hospital for a laparoscopic right nephrectomy. She has had hypertension for 6 years, but there were no abnormal findings in her laboratory results or physical examination. Etomidate $8 \mathrm{mg}$ and succinylcholine $50 \mathrm{mg}$ were given for induction of anesthesia. After confirming the loss of consciousness by entropy and adequate muscle relaxation by 4 twitches on the accelerograph (TOF-watch ${ }^{\circledR}$, Organon Ltd., Dublin, Ireland), we inserted a $7.5 \mathrm{~mm}$-sized poly vinyl chloride with high-volume and low-pressure cuffed endotracheal tube using a direct curved laryngoscope. The tube was inserted about $20 \mathrm{~mm}$ beyond the vocal cords without resistance at the time while looking at the vocal cords, and then, the tube was placed on the incisor teeth at $21 \mathrm{~cm}$. There was no blood in the mouth or airway. Anesthesia was maintained with 1.5-5.8\% desflurane in $\mathrm{O}_{2}$ with $50 \% \mathrm{~N}_{2} \mathrm{O}$ and atracurium. During the 150 minute surgery, her positioning tilted 20 degrees to the left was held. We removed the tube after confirming recovery of consciousness and spontaneous respiration. The endotracheal tube was fixed to the incisor teeth at $21 \mathrm{~cm}$ until its removal. The next day after the removal of the endotracheal tube, she complained of a sore throat, hoarseness, and mild aspiration of food. Two days later, the sore throat and aspiration of food improved, but the hoarseness lasted for 7 days after the removal of the endotracheal tube. Then, an otolaryngologist observed left vocal cord paralysis and right vocal cord edema (Fig. 1). Thus, starting with dexamethasone $10 \mathrm{mg}$ and reducing the dose of the drug gradually for 7 days, we observed relief of the hoarseness and disappearance of the right vocal cord edema. In addition, 30 days after the removal of the endotracheal tube, the laryngograph precision stroboscopy showed normal movement of the left vocal cord.

The causes of unilateral vocal cord paralysis are divided into joint disorders and nerve damage. Inserting an endotracheal tube violently or compressing the arytenoids by using an unsuitable tube for a long time can cause joint disorders such as dislocation or damage of the cricoarytenoid joint and the cricothyroid joint. In such cases, the larynx shows an abnormal arytenoid shape, bleeding, or inflammation. In this case, the possibility of joint damage could be ruled out because the laryngoscopy did not show the above-mentioned aspects on the larynx.

Some causes of nerve damage are an unsuitable position during surgery, compression due to movement of the position, and the high pressure of the endotracheal tube's cuff itself. The anterior branch of the recurrent laryngeal nerve can be compressed between the endotracheal tube's cuff and the lamina of the thyroid cartilage when the cuff is inflated. When the endotracheal tube's cuff inflates, the cuff can compress the anterior branch of the recurrent laryngeal nerve, which causes damage to the nerve. Therefore, the cuff's position of the endotracheal tube should be located at more than $15 \mathrm{~mm}$ below the vocal

Corresponding author: Dong Ai An, M.D., Ph.D., Department of Anesthesiology and Pain Medicine, Kangbuk Samsung Hospital, Sungkyunkwan University School of Medicine, 108, Pyeong-dong, Jongno-gu, Seoul 110-746, Korea. Tel: 82-2-2001-2319, Fax: 82-2-2001-2326, E-mail: andongai@gmail.com

(c) This is an open-access article distributed under the terms of the Creative Commons Attribution Non-Commercial License (http:// creativecommons.org/licenses/by-nc/3.0/), which permits unrestricted non-commercial use, distribution, and reproduction in any medium, provided the original work is properly cited. 

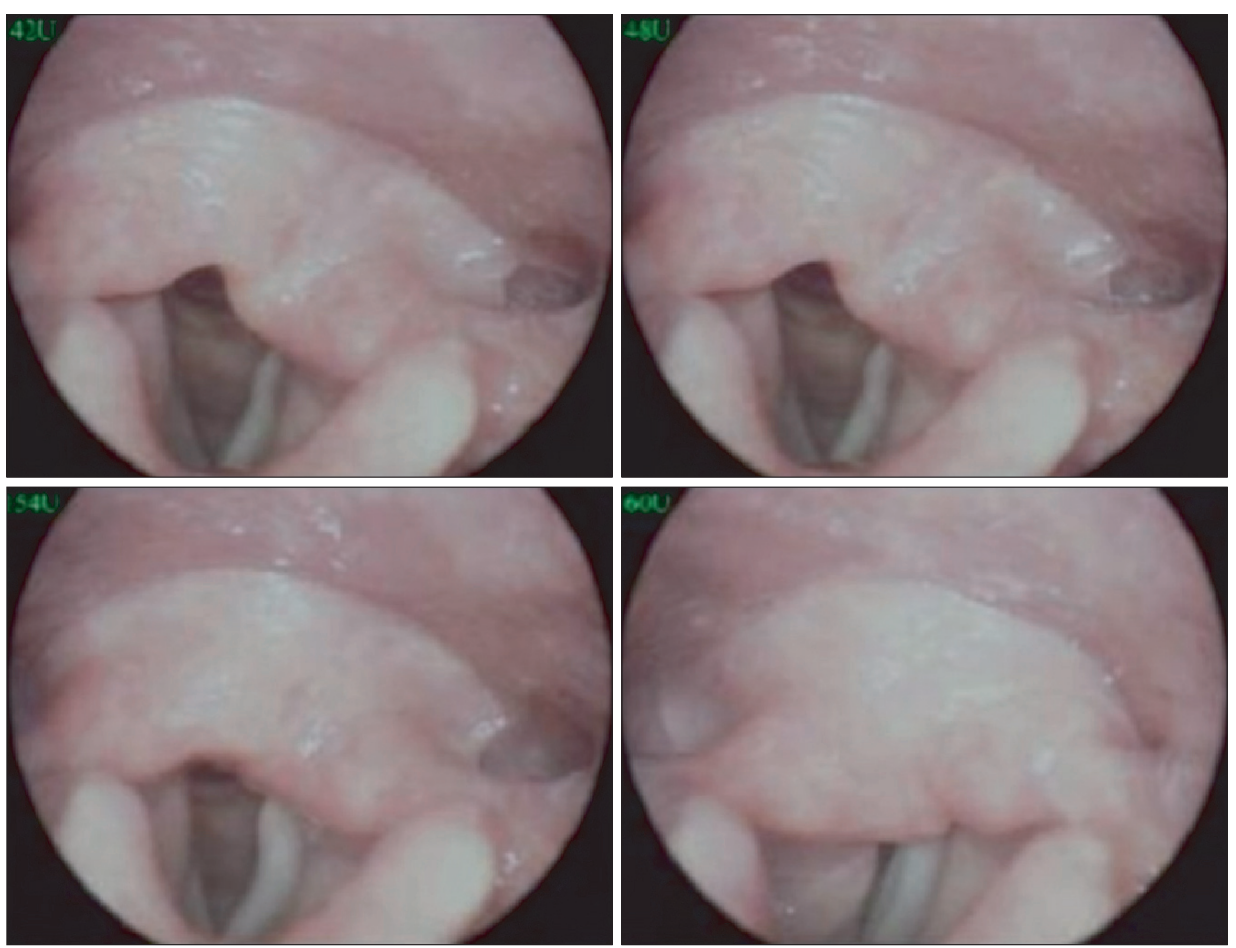

Fig. 1. Laryngograph precision stroboscopy at the time of the vocal cord paralysis. Seven days after extubation, the laryngograph precision stroboscopy showed left vocal cord paralysis at the adduction position and right vocal cord edema.

cords in order to avoid expansion of the cuff in the larynx [2]. Because nitrous oxide diffuses into the cuff more rapidly than nitrogen, intracuff pressure can increase significantly even for a short time of endotracheal intubation. Such increased cuff pressure can cause damage to the mucous membrane of the larynx and the trachea and compress the nerve [3]. Hence, we had to use an endotracheal tube with a high volume-low pressure cuff and monitor the intracuff pressure regularly by using a simple decompression valve.

In this case, where our patient was administered nitrous oxide for 150 minutes and we did not check the intracuff pressure, nerve damage due to the overinflated cuff could not be ruled out.

Kikura et al. [4] have reported that the relative risk of vocal cord paralysis induced by endotracheal intubation increases three-fold in patients aged 50 years or older because of vulner- able laryngeal tissues, and two-fold in patients with chronic diseases such as hypertension or diabetes. Concerning the length of the intubation period, the risk increases to two-fold in patients intubated 3-6 hours, and 15-fold in patients intubated 6 hours or more. In this case, the relative risk of vocal cord paralysis is expected to increase due to the patient being 80 years of age while expected to decrease due an intubation period less than 3 hours.

Unilateral vocal cord paralysis following endotracheal intubation usually resolves spontaneously. However, it can be very dangerous as edema of the vocal cords can cause dyspnea. Thus, prevention of vocal cord paralysis is very important. Wang et al. [5] reported that they gave their patients steroids to prevent dyspnea. Even with a short period of endotracheal intubation especially in elderly patients, paralysis or edema of the vocal cords can occur easily. Therefore, early diagnosis and treatment should be carried out.

\section{References}

1. Miller RD. Anesthesia. 7th ed. Edited by Miller RD, Eriksson LI, Fleisher LA, Wiener-Kronish JP, Young WL: New York, Churchil Livingstone. 2009, p 2458.

2. Benumof JL. Airway Management: Principles and Practice. St. Louis, Mosby. 1996, p 868.

3. Stanley HT, Kawaman R, Graves C. Effects of nitrous oxide on volume and pressure of endotracheal tube cuffs. Anesthesiology 1974; 41: 256-62.

4. Kikura M, Suzuki K, Itagaki T, Takada T, Sato S. Age and comorbidity as risk factors for vocal cord paralysis associated with tracheal intubation. Br J Anaesth 2007; 98: 524-30.

5. Wang CL, Tsai YH, Huang CC, Wu YK, Ye MZ, Chou HM, et al. The role of the cuff leak test in predicting the effects of corticosteroid treatment on postextubation stridor. Chang Gung Med J 2007; 30: 53-61. 